\title{
Beam Energy Dependence of Strange Hadron Production at RHIC
}

\author{
Feng Zhao (for the STAR Collaboration)* \\ University of California, Los Angeles \\ 475 Portola Plaze, Knudsen Hall 5-117 \\ Los Angeles, CA, 90095 \\ E-mail: fengzhao@mail.physics.ucla.edu
}

We report the STAR measurement of $K_{S}^{0}, \Lambda, \Xi, \Omega$ spectra and yields at mid-rapidity $(|y|<0.5)$ in $\mathrm{Au}+\mathrm{Au}$ Collisions at $\sqrt{s_{N N}}=7.7,11.5,19.6,27$, and $39 \mathrm{GeV}$. The measurements of nuclear modification factors $\left(R_{C P}\right)$, anti-baryon to baryon ratios, and baryon-to-meson ratios are presented as a function of $p_{T}$ and beam energy. Physics implications of our measurements on particle production mechanism are also discussed.

8th International Workshop on Critical Point and Onset of Deconfinement, March 11 to 15, 2013

Napa, California, USA

\footnotetext{
* Speaker.
} 


\section{Introduction}

Strangeness production is considered to be a signature of the deconfined quark-gluon matter in the heavy ion collisions, and provides a sensitive probe to the nuclear medium created in the collisions [1]. The mechanism of strangeness production varies with the collision energy. In RHIC $\mathrm{Au}+\mathrm{Au} 200 \mathrm{GeV}$ collisions, it is observed that the nuclear modification factors $\left(R_{C P}\right)$ of baryons and mesons at intermediate $p_{T}$ follow different trends, and the baryon-to-meson ratios are enhanced at intermediate $p_{T}$ range in central collisions [2] [3]. At high $p_{T}$, the $R_{C P}$ is much less than unity. And there is no significant difference between the behaviors of strange hadrons and other light flavor hadrons. Those phenomena may be explained by the partonic energy loss in the medium and the recombination/coalescence model [4] [5] [6] [7] [8], which is an indication of the formation of the strongly coupled quark-gluon plasma (sQGP). At low beam energies, such as AGS and SPS energies, the associate production mechanism seems to dominate the particle production. Our measurement of strange particle yields, $R_{C P}$, anti-baryon to baryon ratios, and baryon-to-meson ratios will reveal how the strangeness production mechanism changes with the beam energy.

STAR has collected high statistics data in years 2010 and 2011 in the RHIC Beam Energy Scan Program at 7.7, 11.5, 19.6, 27, and $39 \mathrm{GeV} \mathrm{Au+Au} \mathrm{collisions.} \mathrm{In} \mathrm{STAR,} \mathrm{we} \mathrm{reconstruct}$ the strange hadrons $\left(K_{S}^{0}, \Lambda, \Xi, \Omega\right)$ using the topology of their weak decay channels [9] [10], $K_{S}^{0} \rightarrow$ $\pi^{+} \pi^{-}$(69.2\% branching ratio), $\Lambda \rightarrow p \pi$ (63.9\% branching ratio), $\Xi \rightarrow \Lambda \pi$ (99.9\% branching ratio), and $\Omega \rightarrow \Lambda K$ (67.8\% branching ratio). The decay daughters $\pi, K, p$ are identified by the Time Projection Chamber (TPC) [11] of the STAR detector system.

\section{Results}

We obtain the $p_{T}$ spectra and yields $(d N / d y)$ at mid-rapidity for $K_{S}^{0}, \Lambda(\bar{\Lambda}), \Xi^{-}\left(\bar{\Xi}^{+}\right)$, and $\Omega^{-}\left(\bar{\Omega}^{+}\right)$after correcting the raw spectra for the geometrical acceptance and the reconstruction efficiencies [12]. The $\Lambda(\bar{\Lambda})$ spectra have been corrected for feed-down contributions from $\Xi$ and $\Xi^{0}$ weak decay. Figure 1 shows the particle yields $(d N / d y)$ at mid-rapidity for $\Lambda, \bar{\Lambda}, \Xi^{-}$, and $\bar{\Xi}^{+}$from the most central (0-5\%) Au+Au collisions at 19.6 and $27 \mathrm{GeV}$. The particle yields at 7.7, 11.5, and $39 \mathrm{GeV}$ are from the Ref. [12]. We also compare the STAR data with NA49 [13], NA57 [14], and STAR high energy data [3] [9] [10] [15]. The $d N / d y$ data are scaled by the number of participants ( $N_{\text {part }}$, named number of wounded nucleons $N_{w}$ in NA49 and NA57), which is estimated with a Monte Carlo Glauber model [16]. The yields of anti-baryons $\left(\bar{\Lambda}, \bar{\Xi}^{+}\right)$increase monotonically with increasing beam energy; $\Lambda$ yields decrease with increasing beam energy at low energies and increase again from $39 \mathrm{GeV}$ to higher energies; while the $\Xi^{-}$yields almost remain the same from 11.5 to $62.4 \mathrm{GeV}$. At low energy, the $\Lambda$ production is enhanced due to the large baryon stopping at mid-rapidity. This effect decreases with increasing beam energy. On the other hand, the QGP dominates the particle production at high energies, and decreases with decreasing beam energy. At intermediate energies, the interplay of both effects may result in a dip in the $\Lambda$ yield as a function of beam energy.

The $R_{C P}$ as a function of $p_{T}$ for $K_{S}^{0}, \Lambda(\bar{\Lambda}), \Xi^{-}\left(\bar{\Xi}^{+}\right), \Omega^{-}\left(\bar{\Omega}^{+}\right)$is shown for beam energies from 7.7 to $39 \mathrm{GeV}$ in Fig.2. The $R_{C P}$ is calculated as the ratio of particle yields at most central collisions $(0-5 \%)$ to yields at peripheral collisions $(40-60 \%)$ scaled by the number of inelastic 


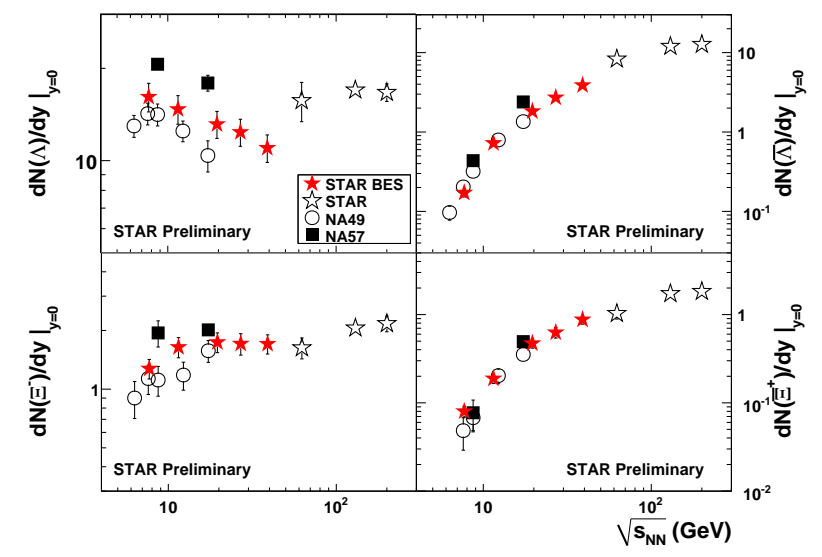

Figure 1: The strange baryon $d N / d y$ at mid-rapidity in most central collisions. The upper left plot is $\Lambda$, the upper right plot is $\bar{\Lambda}$, the lower left plot is $\Xi^{-}$, and the lower right plot is $\bar{\Xi}^{+}$. The rapidity ranges are $|y|<0.5$ for STAR and NA57, $|y|<0.4$ for NA49 $\Lambda(\bar{\Lambda})$ and $|y|<0.5$ for NA49 $\Xi^{-}\left(\bar{\Xi}^{+}\right)$. The centrality bins are $0-5 \%$ for STAR, $0-7 \%$ for NA49 6.3 to $12.3 \mathrm{GeV}$ and $0-10 \%$ for NA49 $17.3 \mathrm{GeV}$, and 0-53\% for NA57.

binary collisions $\left(N_{b i n}\right) . N_{b i n}$ is estimated with the Monte Carlo Glauber model. The $R_{C P}$ data are from Refs. [12] [17]. The strange hadron $R_{C P}$ has a similar behavior in 19.6, 27, and $39 \mathrm{GeV}$. Although the baryon/meson separation is not as significant as in $200 \mathrm{GeV}$ [2] [3], there seems to be a difference between $K_{S}^{0} R_{C P}$ and strange-baryon $R_{C P}$. In lower energies (7.7 and $11.5 \mathrm{GeV}$ ), the $R_{C P}$ of all $K_{S}^{0}, \Lambda, \Xi^{-}$increases with $p_{T}$. We compare the $R_{C P}$ of $K_{S}^{0}$ for different energies in Fig.3. The $R_{C P}$ of $K_{S}^{0}$ increases with decreasing beam energy from 62.4 to $7.7 \mathrm{GeV}$. In addition, at 7.7 and $11.5 \mathrm{GeV}$, the $R_{C P}$ increases monotonically with $p_{T}$, and $R_{C P}$ becomes greater than unity at $p_{T}>2 \mathrm{GeV} / \mathrm{c}$. The change of $R_{C P}$ behavior indicates that the partonic energy loss effect becomes less important at low energies, while the cold nuclear matter effect (Cronin effect) [18] begins to take over.

Figure 4 shows the anti-baryon to baryon ratios $(\bar{B} / B)$ from STAR BES, STAR higher energies and NA49 data. In the statistical thermal model [19], $\ln (\bar{B} / B)$ is proportional to $\left(-2 \times \frac{\mu_{B}}{T}+\Delta S \times\right.$ $\frac{\mu_{S}}{T}$ ), where $\mu_{B}$ is the baryon chemical potential, $\mu_{S}$ is the strangeness chemical potential, $T$ is the chemical freeze-out temperature, and $\Delta S$ is the difference between the strange numbers of $\bar{B}$ and $B$. The ratios increase with increasing beam energy, due to the decreasing $\mu_{B}$, and the increasing $T$. At the same energy, the $\bar{\Omega}^{+} / \Omega^{-}$is larger than the $\bar{\Xi}^{+} / \Xi^{-}$, and the $\bar{\Xi}^{+} / \Xi^{-}$is larger than the $\bar{\Lambda} / \Lambda$, due to the strangeness chemical potential $\left(\mu_{S}\right)$. At low energies, the coefficient of proportionality $\left(\mu_{S} / T\right)$ plays a significant role, making the difference among $\bar{\Omega}^{+} / \Omega^{-}, \bar{\Xi}^{+} / \Xi^{-}$and $\bar{\Lambda} / \Lambda$ large.

Figure 5 shows the $\Lambda / K_{S}^{0}$ ratios from most central collisions (0-5\%) at 7.7 to $62.4 \mathrm{GeV}$. The ratio increases with decreasing beam energy, due to the large cross-section of $\Lambda$ in low energy. From 19.6 to $62.4 \mathrm{GeV}$, there is an enhancement in the ratio at $p_{T}$ around $2.7 \mathrm{GeV} / \mathrm{c}$. Due to the limited statistics at 7.7 and $11.5 \mathrm{GeV}$, the $\Lambda / K_{S}^{0}$ ratios cannot reach high $p_{T}$ range as higher energies. So it seems $\Lambda / K_{S}^{0}$ ratios increase monotonically with increasing $p_{T}$ at 7.7 and $11.5 \mathrm{GeV}$. Further study with increased statistics is necessary to draw a conclusion. 

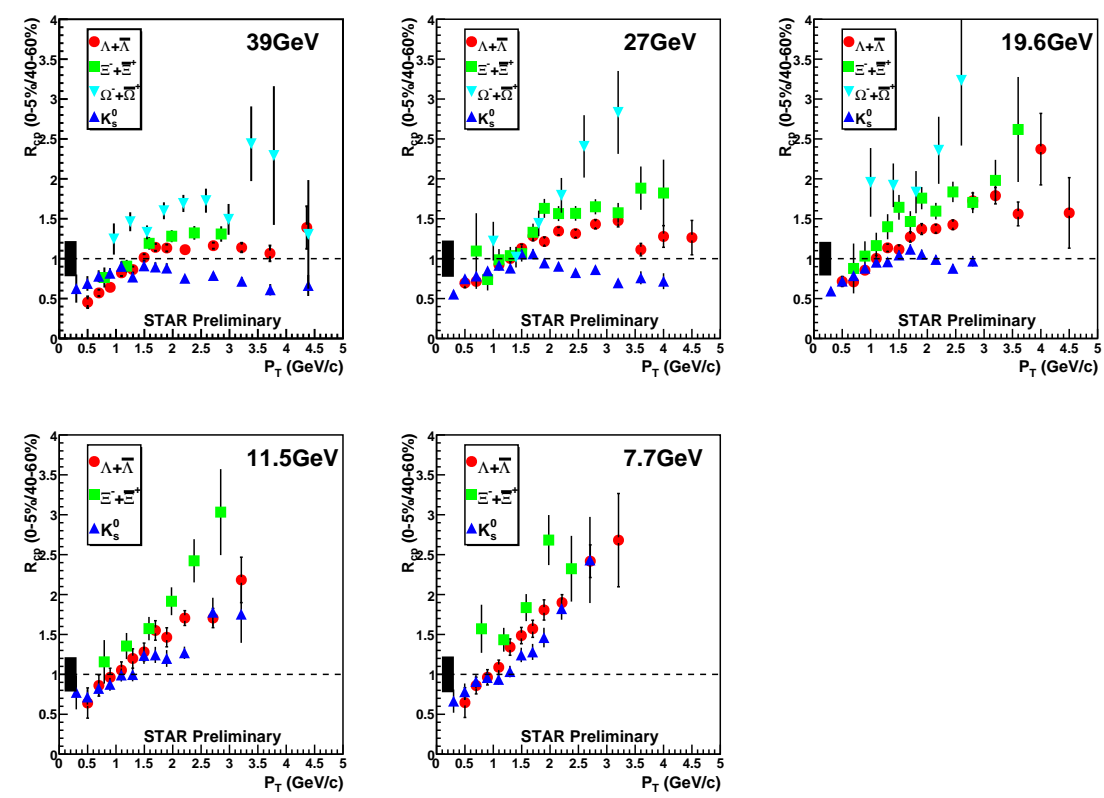

Figure 2: The nuclear modification factor $\left(R_{C P}\right)$, as a function of $p_{T}$ for $K_{S}^{0}, \Lambda(\bar{\Lambda})$, and $\Xi^{-}\left(\bar{\Xi}^{+}\right.$at $|y|<0.5$ from $\mathrm{Au}+\mathrm{Au}$ collisions at $\sqrt{s_{N N}}$ from 39 to $7.7 \mathrm{GeV}$. Errors are statistical only.

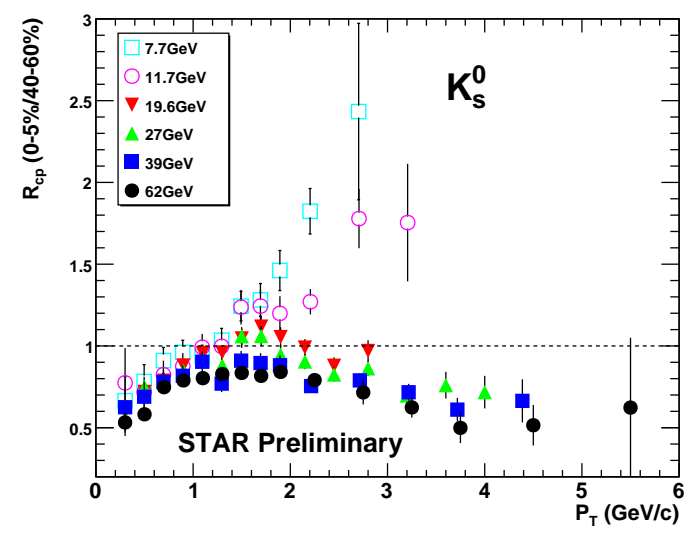

Figure 3: The $R_{C P}$ of $K_{S}^{0}$ as a function of $p_{T}$ from Au+Au collisions at $\sqrt{s_{N N}}$ from 7.7 to $62.4 \mathrm{GeV}$. Errors are statistical only.

\section{Summary}

In summary, we have presented the strange particle yields $(d N / d y)$ at mid-rapidity, as well as the particle $R_{C P}$ and $\Lambda / K_{S}^{0}$ ratios. The results indicate that the QGP dominates the particle production at high beam energies. At low energies, the associate production dominates the particle production, and the cold nuclear effect is significant. 


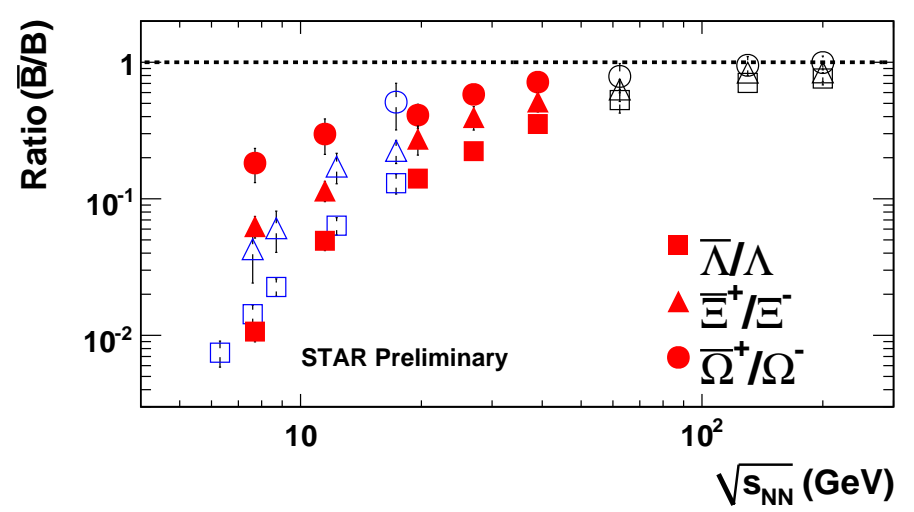

Figure 4: The anti-baryon to baryon ratios as a function of $\sqrt{S_{N N}}$ at most central Au+Au collisions from $\mathrm{STAR}$ and $\mathrm{Pb}+\mathrm{Pb}$ collisions from NA49. Solid symbols are STAR BES data, open symbols are STAR higher energies (greater than $62.4 \mathrm{GeV}$ ) data and NA49 data.

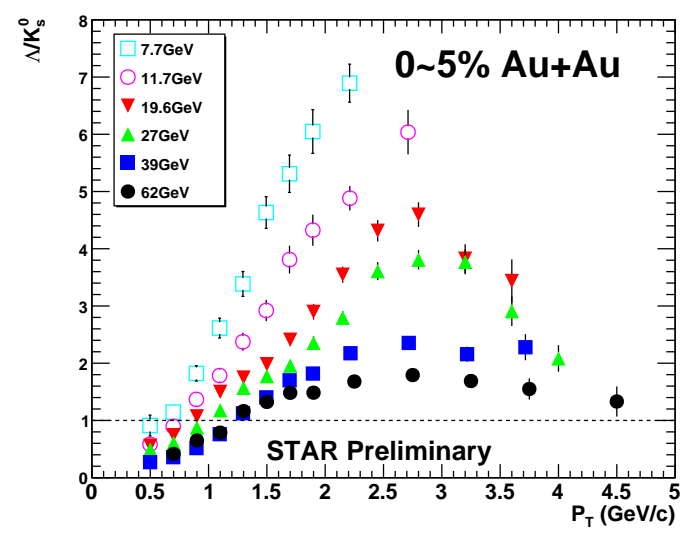

Figure 5: The $\Lambda / K_{S}^{0}$ ratio as a function of $p_{T}$ from $\mathrm{Au}+\mathrm{Au}$ collisions at most central (0-5\%) $\mathrm{Au}+\mathrm{Au}$ collisions at $\sqrt{s_{N N}}$ from 7.7 to $62.4 \mathrm{GeV}$. Errors are statistical only.

\section{References}

[1] J. Rafelski, B. Muller, Phys. Rev. Lett. 48 (1982) 1066

[2] STAR Collaboration, M.M. Aggarwal et al., arXiv:nucl-ex/1007.2613v1

[3] STAR Collaboration, J. Adams et al., Phys. Rev. Lett. 98 (2007) 062301

[4] M.A.C. Lamont (for the STAR Collaboration), J. Phys. Conf. Ser. 50 (2006) 192

[5] R.C. Hwa, C.B. Yang, Phys. Rev. C 66 (2002) 025205

[6] V. Greco, C.M. Ko, P. Levai, Phys. Rev. Lett. 90 (2003) 202302

[7] R.J. Fries, B. Muller, C. Nonaka, S.A. Bass, Phys. Rev. Lett. 90 (2003) 202303

[8] D. Molnar, S.A. Voloshin, Phys. Rev. Lett. 90 (2003) 092301 
[9] STAR Collaboration, C. Adler et al., Phys. Rev. Lett. 89 (2002) 092301

[10] STAR Collaboration, J. Adams et al., Phys. Rev. Lett. 92 (2004) 182301

[11] M. Anderson et al., Nucl. Instrum. Meth. A 499 (2003) 659

[12] X. Zhu (for the STAR Collaboration), Acta Phys. Polon. Supp. 5 (2012) 213

[13] NA49 Collaboration, C. Alt et al., Phys. Rev. C 78 (2008) 034918

[14] NA57 Collaboration, F. Antinori et al., Phys. Lett. B 595 (2004) 68

[15] STAR Collaboration, M.M. Aggarwal et al., Phys. Rev. C 83 (2011) 024901

[16] STAR Collaboration, B.I. Abelev et al., Phys. Rev. C 79 (2009) 034909

[17] X. Zhang (for the STAR Collaboration), arXiv:nucl-ex/1305.4476v1

[18] J.W. Cronin et al., Phys. Rev. D 11 (1975) 3105

[19] J. Cleymans, arXiv:nucl-th/9704046v1 